\title{
For Distinguishing Conjugate Hidden Subgroups, the Pretty Good Measurement is as Good as it Gets
}

\author{
Cristopher Moore \\ moore@cs.unm.edu \\ Department of Computer Science \\ University of New Mexico
}

\author{
Alexander Russell \\ acr@cse.uconn.edu \\ Department of Computer Science and Engineering \\ University of Connecticut
}

May 19, 2005

\begin{abstract}
Recently Bacon, Childs and van Dam showed that the "pretty good measurement" (PGM) is optimal for the Hidden Subgroup Problem on the dihedral group $D_{n}$ in the case where the hidden subgroup is chosen uniformly from the $n$ involutions. We show that, for any group and any subgroup $H$, the PGM is the optimal one-register experiment in the case where the hidden subgroup is a uniformly random conjugate of $H$. We go on to show that when $H$ forms a Gel'fand pair with its parent group, the PGM is the optimal measurement for any number of registers. In both cases we bound the probability that the optimal measurement succeeds. This generalizes the case of the dihedral group, and includes a number of other examples of interest.
\end{abstract}

\section{The Hidden Conjugate Problem}

Consider the following special case of the Hidden Subgroup Problem, called the Hidden Conjugate Problem in [16]. Let $G$ be a group, and $H$ a non-normal subgroup of $G$; denote conjugates of $H$ as $H^{g}=g^{-1} H g$. Then we are promised that the hidden subgroup is $H^{g}$ for some $g$, and our goal is to find out which one.

The usual approach is to prepare a uniform superposition over the group, entangle the group element with a second register by calculating or querying the oracle function, and then measure the oracle function. This yields a uniform superposition over a random left coset of the hidden subgroup,

$$
\left|c H^{g}\right\rangle=\frac{1}{\sqrt{|H|}} \sum_{h \in H^{g}}|c h\rangle
$$

Rather than viewing this as a pure state where $c$ is random, we may treat this as a classical mixture over left cosets, giving the mixed state with density matrix

$$
\rho_{g}=\frac{1}{|G|} \sum_{c \in G}\left|c H^{g}\right\rangle\left\langle c H^{g}\right|
$$

We then wish to find a positive operator-valued measurement (POVM) to identify $g$.

A POVM consists of a set of positive measurement operators $\left\{E_{i}\right\}$ that obey the completeness condition

$$
\sum_{i} E_{i}=\mathbb{1} .
$$

If we are trying to distinguish $\ell$ density matrices $\left\{\rho_{i} \mid 1 \leq i \leq \ell\right\}$, and if $\rho_{i}$ is chosen with probability $p_{i}$, the probability that the POVM gives the right answer is

$$
P_{\text {success }}=\sum_{i} p_{i} \operatorname{tr} E_{i} \rho_{i} .
$$


A theorem of Yuen, Kennedy and Max [22] and Holevo [9] states that $P_{\text {success }}$ is maximized if and only if the following conditions hold for every $i$ :

$$
\begin{gathered}
\left(\sum_{j} p_{j} \rho_{j} E_{j}-p_{i} \rho_{i}\right) E_{i}=0, \\
\sum_{j} p_{j} \rho_{j} E_{j}=\sum_{j} p_{j} E_{j} \rho_{j}, \text { and } \\
\sum_{j} p_{j} \rho_{j} E_{j} \geq p_{i} \rho_{i},
\end{gathered}
$$

where we write $A \geq B$ if $A-B$ is positive semidefinite. These conditions come from recognizing that maximizing $P_{\text {success }}$ subject to the completeness condition (1.2) gives a semidefinite program. Ip [11] used this fact to show that Shor's algorithm is optimal for the hidden subgroup problem on the cyclic group $\mathbb{Z}_{n}$.

In a beautiful recent paper, Bacon, Childs and van Dam [1] consider the hidden conjugate problem for the dihedral group $D_{n}$ where $H$ is an order-2 subgroup; that is, when the hidden subgroup consists of the identity and one of the $n$ involutions. They consider entangled measurements over multiple registers each of which contains a coset state, and show that the so-called "pretty good measurement" [7], defined below, is in fact optimal for any number of registers. They then show that this optimal measurement is related to random cases of the Subset Sum problem (see also Regev [18]). Other prior work includes Eldar, Megretski, and Verghese [4], who showed that the PGM is optimal for certain families of density matrices related by group symmetries; see references in that paper for some other cases for which the PGM is optimal.

In this paper we point out that for any subgroup $H$ of a group $G$, the pretty good measurement is the optimal one-register experiment for finding a hidden conjugate of $H$ when all conjugates are equally likely. We then write a general expression for the probability $P_{\text {success }}$ that this measurement identifies the hidden conjugate in a single experiment on a coset state. We then go on to show that when $G$ and $H$ form a Gel'fand pair, the pretty good measurement is in fact the optimal measurement on any number of registers. This recovers the optimality result of [1] as a special case, and establishes optimal measurements for a variety of other interesting group-subgroup pairs, including the subgroups of the affine groups studied in [16].

We use the machinery of representation theory; we refer the reader to $[6,20]$ or to the review in our paper [14] for an introduction and for notation.

\section{The one-register case}

\subsection{The pretty good measurement is optimal}

The pretty good measurement (PGM), also known as the least squares measurement, is defined as follows [7]. Given a set of density matrices $\rho_{i}$ with associated probabilities $p_{i}$, let

$$
M=\sum_{i} p_{i} \rho_{i} .
$$

Then the PGM associated with this family of mixed states is $\left\{E_{i}\right\}$, where the measurement operator $E_{i}$ is defined as

$$
E_{i}=p_{i} M^{-1 / 2} \rho_{i} M^{-1 / 2}
$$

where the inverse $M^{-1 / 2}$ is defined on the image of $M$; that is, $M^{-1 / 2}$ is the unique positive operator such that $\left(M^{-1 / 2}\right)^{2} M$ is the projection operator $\Pi$ onto the image of $M$. If $M$ has full rank, it is easy to see that this choice of $\left\{E_{i}\right\}$ satisfies the completeness condition (1.2); if the image of $M$ is a proper subspace, we satisfy the completeness condition by adding an additional measurement which projects onto its orthogonal complement. 
We will show that the optimality conditions (1.4), (1.5), and (1.6) hold for the PGM for the family of density matrices $\left\{\rho_{g}\right\}$ defined above when endowed with the uniform distribution $p_{g}=1 /|G|$. First we derive the structure of the PGM. Observe that the mixed state $\rho_{g}=|G|^{-1} \sum_{c}\left|c H^{g}\right\rangle\left\langle c H^{g}\right|$ has the property that

$$
\rho_{g}^{2}=\frac{|H|}{|G|} \rho_{g}
$$

so that $\rho_{g}$ is a projection operator scaled by the constant $|H| /|G|$ (this follows from the fact that the uniform distribution on any subgroup is its own square under convolution). As $\operatorname{tr} \rho_{g}=1$, we must have rk $\rho_{g}=|G| /|H|$, the index of $H$ in $G$.

Note that $\rho_{g}$ commutes with the left action of $G$, since it is "symmetrized" over all left cosets. By Schur's lemma, $\rho_{g}$ is block diagonal, with blocks corresponding to the decomposition of $\mathbb{C}[G]$ into bi-invariant spaces. Furthermore, the block corresponding to the irreducible representation $\sigma$ has form $A_{\sigma} \otimes \mathbb{1}_{d_{\sigma}}$, where $\mathbb{1}_{d_{\sigma}}$ acts within each left-invariant space. Recalling (2.2), $A_{\sigma}$ is a rescaled projection operator, and we may write $\rho_{g}=\oplus_{\sigma \in \widehat{G}} \rho_{g}^{\sigma}$ where

$$
\rho_{g}^{\sigma}=\frac{|H|}{|G|} \pi_{g}^{\sigma} \otimes \mathbb{1}_{d_{\sigma}} ;
$$

here $\pi_{g}^{\sigma}$ is the projection operator

$$
\pi_{g}^{\sigma}=\frac{1}{|H|} \sum_{h \in H^{g}} \sigma(h)
$$

Since rk $\pi_{g}^{\sigma}$ does not depend on $g$, we denote it simply as rk $\pi^{\sigma}$. Then the following lemma describes the structure of the PGM for $\left\{\rho_{g}\right\}$. Since $\rho_{g}=\rho_{h}$ whenever $g$ and $h$ are in the same coset of the normalizer $\operatorname{Norm}(H)=\left\{g \mid H^{g}=H\right\}$, without loss of generality we assume that the POVM gives a uniformly random element of some coset of $\operatorname{Norm}(H)$, and we count it as having succeeded if it gives an element of the correct coset. Thus we will multiply $P_{\text {success }}$ by the index of $\operatorname{Norm}(H)$ below.

Lemma 1. For the family $\left\{\rho_{g}\right\}$ of density matrices corresponding to random left cosets of conjugate subgroups $H^{g}$ with the uniform distribution on $g$, the pretty good measurement operators $E_{g}$ are given by

$$
E_{g}=\bigoplus_{\sigma \in \widehat{G}} E_{g}^{\sigma}
$$

and, for each $\sigma \in \widehat{G}$,

$$
E_{g}^{\sigma}=\frac{d_{\sigma}}{|G| \boldsymbol{r k} \pi^{\sigma}} \pi_{g}^{\sigma} \otimes \mathbb{1}_{d_{\sigma}}
$$

where $\pi_{g}^{\sigma}$ is defined as in (2.3).

Proof. For each $x \in G$, let $L_{x}$ and $R_{x}$ denote the unitary operators that carry out left and right group multiplication by $x$. Note that left cosets are mapped to each other by $L_{x}$ and, in particular, $|c H\rangle=L_{c}|H\rangle$. Note furthermore that $R_{x}$ maps left cosets of one conjugate onto left cosets of another conjugate, e.g., $\left|H^{g}\right\rangle=R_{g}\left|g^{-1} H\right\rangle$, and that $L_{x}$ commutes with $R_{y}$ for all $x, y$.

We have $p_{g}=1 /|G|$ for all $g$. Now if we write

$$
\begin{aligned}
M & =\frac{1}{|G|} \sum_{g} \rho_{g}=\frac{1}{|G|^{2}} \sum_{c, g}\left|c H^{g}\right\rangle\left\langle c H^{g}\left|=\frac{1}{|G|^{2}} \sum_{c, g}\right| c H g\right\rangle\langle c H g| \\
& =\frac{1}{|G|^{2}} \sum_{c, g} L_{c} R_{g}|H\rangle\langle H| R_{g}^{\dagger} L_{c}^{\dagger}
\end{aligned}
$$

(where in the third equality we replace $c g^{-1}$ with $c$ ), we see that $M$ commutes with $L_{x}$ and $R_{x}$ for all $x \in G$. That is, summing over both the left coset and the choice of conjugate "symmetrizes" $M$ on both the left and the right. It follows by Schur's lemma that $M$ takes the form

$$
M=\bigoplus_{\sigma \in \widehat{G}} M^{\sigma}
$$


where $M^{\sigma}$ is a scalar multiple of the identity operator for each $\sigma$. As

$$
M_{\sigma}=\frac{1}{|G|} \sum_{g} \rho_{g}^{\sigma}=\frac{|H|}{|G|^{2}} \sum_{g} \pi_{g}^{\sigma} \otimes \mathbb{1}_{d_{\sigma}}
$$

by taking traces we conclude that

$$
M^{\sigma}=\left(\frac{|H|}{|G|} \frac{\mathbf{r k} \pi^{\sigma}}{d_{\sigma}}\right) \mathbb{1}_{d_{\sigma}^{2}} .
$$

Similarly, $\rho_{g}$ is block-diagonal, as it commutes with $L_{x}$ (though not with $R_{x}$ unless $H$ is normal). Therefore, $M$ commutes with $\rho_{g}$ for each $g$, and (2.1) becomes

$$
E_{g}=\frac{1}{|G|} M^{-1} \rho_{g}
$$

giving, in each irreducible block,

$$
E_{g}^{\sigma}=\frac{d_{\sigma}}{|H| \mathbf{r k} \pi^{\sigma}} \rho_{g}^{\sigma}=\frac{d_{\sigma}}{|G| \mathbf{r k} \pi^{\sigma}} \pi_{g}^{\sigma} \otimes \mathbb{1}_{d_{\sigma}}
$$

which completes the proof.

We now give our proof that the PGM is optimal for the hidden conjugate problem for any $G$ and $H$. This follows simply from the fact that $M$, and therefore $E_{g}$, commutes with $\rho_{g}$ for each $g$.

Theorem 2. For the family $\left\{\rho_{g}\right\}$ of density matrices corresponding to random left cosets of conjugate subgroups $H^{g}$ with the uniform distribution on $g$, the pretty good measurement $\left\{E_{g}\right\}$ optimizes the probability of correctly measuring $g$.

Proof. Since the $E_{g}$ and $\rho_{g}$ are block-diagonal (according to the same decomposition of $\mathbb{C}[G]$ ), it suffices to confirm the optimality criteria (1.4), (1.5), and (1.6) in each block, i.e., for each $\sigma \in \widehat{G}$.

First, (1.5) holds trivially since $E_{g}^{\sigma}$ is proportional to $\rho_{g}^{\sigma}$ for each $g$, and so commutes with it. As for condition (1.6), observe that from (2.2) and (2.7) we have

$$
\rho_{g}^{\sigma} E_{g}^{\sigma}=\frac{d_{\sigma}}{|G| \mathbf{r k} \pi^{\sigma}} \rho_{g}^{\sigma}
$$

and so for any $h \in G$, recalling that $p_{g}=1 /|G|$ for all $g$, we have

$$
\frac{1}{|G|} \sum_{g} \rho_{g}^{\sigma} E_{g}^{\sigma}=\frac{d_{\sigma}}{|G| \mathbf{r k} \pi^{\sigma}} M^{\sigma}=\frac{|H|}{|G|^{2}}\left(\mathbb{1}_{d_{\sigma}} \otimes \mathbb{1}_{d_{\sigma}}\right) \geq \frac{|H|}{|G|^{2}}\left(\pi_{h}^{\sigma} \otimes \mathbb{1}_{d_{\sigma}}\right)=\frac{1}{|G|} \rho_{h}
$$

since $\mathbb{1}_{d_{\sigma}} \geq \pi_{h}^{\sigma}$. Finally, for any $h \in G$

$$
\left(\sum_{g} \rho_{g}^{\sigma} E_{g}^{\sigma}-\rho_{h}^{\sigma}\right) E_{h}^{\sigma}=\frac{|H|}{|G|}\left[\left(\mathbb{1}_{d_{\sigma}}-\pi_{h}^{\sigma}\right) \otimes \mathbb{1}_{d_{\sigma}}\right] E_{h}^{\sigma}=\frac{|H| d_{\sigma}}{|G|^{2} \mathbf{r k} \pi^{\sigma}}\left[\left(\mathbb{1}_{d_{\sigma}}-\pi_{h}^{\sigma}\right) \pi_{h}^{\sigma}\right] \otimes \mathbb{1}_{d_{\sigma}}=0
$$

since $(\mathbb{1}-\pi) \pi=0$ for any projection operator $\pi$. Again recalling $p_{g}=1 /|G|$ establishes (1.4) and completes the proof.

Note that Lemma 1 and Theorem 2 imply that, as pointed out before $[11,13,14]$, the optimal measurement consists of first measuring the representation name $\sigma$, and then performing an additional measurement $M_{g}^{\sigma}$ inside the bi-invariant space corresponding to $\sigma$. 


\subsection{Partial measurements}

Suppose that rather than trying to identify the conjugate exactly, we wish to learn some partial information about it. To learn one bit, for instance, we would divide the set of conjugates into two equal subsets, and combine the $\rho_{g}$ into two mixed states $\rho_{0}$ and $\rho_{1}$ consisting of mixtures of those in the two subsets. The next theorem shows that the PGM is optimal for any such partial measurement as long as each subset of the set of conjugates has probability proportional to its size.

Theorem 3. Let $C(H)=\left\{H^{g}\right\}$ be partitioned into disjoint sets $C_{i}, 1 \leq i \leq \ell$. Let

$$
\rho_{i}=\frac{1}{\left|C_{i}\right|} \sum_{g: H^{g} \in C_{i}} \rho_{g}
$$

where $\rho_{g}$ is as in (1.1), and let $p_{i}=\left|C_{i}\right| /|C(H)|$. Then the pretty good measurement for the family $\left\{\rho_{i}\right\}$ with probabilities $p_{i}$ is the family of operators $\left\{E_{i}\right\}$

$$
E_{i}=\sum_{g: H^{g} \in C^{i}} E_{g}
$$

where $E_{g}$ is given by Lemma 1; this measurement is optimal.

Proof. The proof is exactly the same as that of Lemma 1 and Theorem 2, except that for each $i$ we sum over the $g$ with $H^{g} \in C_{i}$.

\subsection{The probability of success}

As a corollary to Theorem 2, we can determine the optimal success probability. Let $C(H)$ denote the set of conjugates of $H$. For a group $G$ the Plancherel measure is the probability distribution on $\widehat{G}$ assigning $\sigma \in \widehat{G}$ the probability $d_{\sigma}^{2} /|G|$; this is the fraction, dimensionwise, of $\mathbb{C}[G]$ consisting of the bi-invariant subspace corresponding to $\sigma$. For a set $S \subset \widehat{G}$, we let $\operatorname{Planch}(S)$ denote the probability of observing an element of $S$ according to the Plancherel measure. We remark that the Plancherel measure is precisely the probability distribution obtained by performing weak Fourier sampling when the hidden subgroup is trivial, since in that case the state $\rho$ is completely mixed.

Given a subgroup $H$ of $G$, let $S_{H} \subseteq \widehat{G}$ denote the set of irreducible representations for which the projection operator $\pi_{H}^{\sigma}=|H|^{-1} \sum_{h \in H} \bar{\sigma}(h)$ is nonzero, and let $H_{G}=\cap_{g} H^{g}$ denote the largest normal subgroup contained in $H$. Then the following theorem gives the success probability of the optimal oneregister experiment.

Theorem 4. Given a group $G$ and a subgroup $H$, the probability that the optimal single-register measurement correctly identifies a uniformly random conjugate of $H$ is

$$
P_{\text {success }}=\frac{|H|}{|C(H)|} \operatorname{Planch}\left(S_{H}\right) \leq \frac{|H|}{|C(H)| \cdot\left|H_{G}\right|} .
$$

Proof. Let us say that $g \sim g^{\prime}$ if $H^{g}=H^{g^{\prime}}$ (and so $\rho_{g}=\rho_{g^{\prime}}$ ). Then $P_{\text {success }}$ is the expectation

$$
P_{\text {success }}=\operatorname{Exp}_{g} \sum_{g^{\prime} \sim g} \operatorname{tr} E_{g^{\prime}} \rho_{g}
$$

where $g$ is selected uniformly in $G$. Since $g \sim g^{\prime}$ if and only if $g$ and $g^{\prime}$ are in the same (right) coset of the normalizer $\operatorname{Norm}(H)=\left\{g \mid H^{g}=H\right\}$, we have

$$
P_{\text {success }}=|\operatorname{Norm}(H)| \cdot \operatorname{Exp}_{g} \operatorname{tr} E_{g} \rho_{g}=|\operatorname{Norm}(H)| \cdot \operatorname{Exp}_{g} \sum_{\sigma} \operatorname{tr} E_{g}^{\sigma} \rho_{g}^{\sigma} .
$$


Considering (2.3) and (2.8), we have

$$
\operatorname{tr} E_{g}^{\sigma} \rho_{g}^{\sigma}=\frac{|H| d_{\sigma}^{2}}{|G|^{2}}=\frac{|H|}{|G|} \operatorname{Planch}(\sigma),
$$

but only for those $\sigma$ where $\rho_{g}^{\sigma}$ and $E_{g}^{\sigma}$ are nonzero, i.e., those for which $\mathbf{r k} \pi^{\sigma}>0$. Thus, we conclude that

$$
P_{\text {success }}=\frac{|\operatorname{Norm}(H)||H|}{|G|} \cdot \sum_{\sigma \in S_{H}} \operatorname{Planch}(\sigma)=\frac{|H|}{|C(H)|} \cdot \operatorname{Planch}\left(S_{H}\right)
$$

where we recall that $|C(H)|=|G| /|\operatorname{Norm}(H)|$.

Now, note that for any subgroup $K \subseteq H$, we have $S_{H} \subseteq S_{K}$ since any $\sigma$ that annihilates $K$ also annihilates $H$. In addition, if $K$ is normal, recall that for any $\sigma$ we have either $\pi_{K}^{\sigma}=0$ or $\pi_{K}^{\sigma}=\mathbb{1}$. It follows that $\sum_{\sigma \in S_{K}} d_{\sigma}^{2}=\sum_{\sigma \in S_{K}} d_{\sigma} \mathbf{r k} \pi_{K}^{\sigma}=\mathbf{r k} \pi_{K}^{R}$ where $R$ is the regular representation, and since $\mathbf{r k} \pi_{K}^{R}=|G| /|K|$ we have $\operatorname{Planch}\left(S_{K}\right)=1 /|K|$. Thus $\operatorname{Planch}\left(S_{H}\right) \leq 1 /\left|H_{G}\right|$, completing the proof of (2.9).

Note that if we observe any $\sigma \notin S_{H}$, we know that the promise that the hidden subgroup is a conjugate of $H$ has been violated. Thus, as in [1], if we are promised that the hidden subgroup is either trivial or a conjugate of $H$, we can complete the PGM with an additional measurement operator $M_{0}$ that projects onto the orthogonal complement of $S_{H}$, and conclude that the hidden subgroup is trivial if we observe the outcome $M_{0}$.

It is interesting to compare Theorems 2 and 4 with known results on the hidden subgroup problem. For the dihedral group $D_{n}$ where $H$ is an order- 2 subgroup, there are $n$ conjugates, and $S_{H}$ consists of all of $\widehat{G}$ except for the sign representation. Thus we have

$$
P_{\text {success }}=\frac{2}{n}\left(1-\frac{1}{2 n}\right) \text {. }
$$

On the other hand, for the affine group $A_{p}$, the maximal subgroup $H=\mathbb{Z}_{p}^{*}$ has $p$ conjugates, and $S_{H}$ includes all but the $p-2$ nontrivial one-dimensional representations. This gives

$$
P_{\text {success }}=\frac{p-1}{p}\left(1-\frac{p-2}{p(p-1)}\right)=1-\frac{2(p-1)}{p^{2}} .
$$

Indeed, Moore, Rockmore, Russell and Schulman [16] gave an explicit algorithm using a von Neumann measurement that succeeds with constant probability. This algorithm can easily be modified to carry out the optimal POVM in polynomial time; see also Bacon, Childs and van Dam [2].

Now let us consider the case of the hidden subgroup problem relevant to Graph Isomorphism in the case of two rigid, connected graphs of size $n / 2$. Here $G=S_{n}$ and $H$ is the order-2 subgroup consisting of $n / 2$ disjoint transpositions,

$$
H=\{1,(12)(34) \cdots(n-1 n)\}
$$

of which there are $(n-1)$ !! conjugates, one for each perfect matching of $n$ items. Using lemmas proved in [14], it is easy to show that for almost all representations $\sigma$ (with respect to the Plancherel distribution) we have $\mathbf{r k} \pi^{\sigma}=(1 \pm o(1)) d_{\sigma} / 2$, so $\operatorname{Planch}\left(S_{H}\right)=1-o(1)$. Thus we have

$$
P_{\text {success }}=\frac{2}{(n-1) ! !}(1-o(1))=n^{-n / 2} e^{O(n)}
$$

This can be generalized to other conjugacy classes using general character bounds due to Roichman [19]; see also Kempe and Shalev [12].

However, it should be emphasized that the fact that $P_{\text {success }}$ is exponentially small does not mean that we need an exponential number of single-register experiments to solve the hidden conjugate problem. In particular, Ettinger and Høyer [5] showed that a polynomial number (i.e., $O(\log |G|)=O(\log n)$ ) of singleregister experiments is enough to determine, information-theoretically, an involution in $D_{n}$. Thus our results here do not subsume the results of Moore, Russell and Schulman [14] and Moore and Russell [15], who showed that it takes an exponential number of single-register experiments, or a super-polynomial number of tworegister experiments, to obtain even a single bit of information about the conjugate of $H$ in $S_{n}$. 


\section{Multiregister measurements and Gel'fand pairs}

For the multiregister experiment, we view states as elements of the Hilbert space $\mathbb{C}\left[G^{k}\right]=\mathbb{C}[G]^{\otimes k}$. We now have a random left coset of the subgroup $H^{k} \subset G^{k}$, and the corresponding mixed state is

$$
\boldsymbol{\rho}_{g}=\rho_{g}^{\otimes k}=\frac{1}{|G|^{k}} \sum_{\mathbf{c} \in G^{k}}\left|\mathbf{c}\left(H^{g}\right)^{k}\right\rangle\left\langle\mathbf{c}\left(H^{g}\right)^{k}\right| .
$$

Since $\rho_{g}^{\otimes k}$ is symmetrized over left cosets, it commutes with left multiplication in $G^{k}$. Thus by Schur's lemma it is block-diagonal, where each block corresponds to a representation $\boldsymbol{\sigma}=\sigma_{1} \otimes \cdots \otimes \sigma_{k}$ of $G^{k}$, and each $\sigma_{i}$ is an irreducible representation of $G$. Indeed, in a given such block we can write

$$
\boldsymbol{\rho}_{g}^{\sigma}=\rho_{g}^{\sigma_{1}} \otimes \cdots \otimes \rho_{g}^{\sigma_{k}} .
$$

The situation in the multiregister case is complicated by the fact that, unlike the one-register case, $M$ does not generally commute with $\boldsymbol{\rho}_{g}$. Indeed, they do not commute even for the two-register case in the dihedral group. We note in passing that they do commute in a few special cases: for instance, when $H$ is generated by an involution that commutes with its conjugates. However, this is not a very interesting case, since then $H$ and its conjugates generate an Abelian subgroup $K \subset G$, and we can distinguish them by solving the hidden subgroup problem on $K$.

However, we can still prove that the PGM is optimal in the case that $G$ and $H$ form a Gel'fand pair; we review the definition here, and also refer the reader to [21] for an introduction. Given a group $G$ and a subgroup $H$, let $\mathcal{B}=\mathcal{B}_{H}(G)$ denote the collection of functions $f: G \rightarrow \mathbb{C}$ that are invariant under both left and right multiplication by $H$, i.e., such that $f(h g)=f(g)=f(g h)$ for all $g \in G$ and $h \in H$. This collection of bi-invariant functions forms a natural algebra under convolution, and $\mathcal{B}$ can be identified with the subalgebra of $\mathbb{C}[G]$ generated by elements corresponding to double cosets, $H g H=\left(\sum_{h \in H} h\right) \cdot g \cdot\left(\sum_{h \in H} h\right)$. Then the following criteria are equivalent, and the pair $(G, H)$ is said to be Gel'fand if any of them hold:

1. $\mathcal{B}$ is commutative.

2. The induced representation $\operatorname{Ind}_{H}^{G} \mathbb{1}$ contains no more than one copy of any particular $\sigma \in \widehat{G}$.

3. For any $\sigma \in \widehat{G}$ and any $f \in \mathcal{B}$, the Fourier transform $\hat{f}(\sigma)=\sum_{g} f(g) \sigma(g)$ has rank at most one.

The third criterion is the one most relevant to our analysis. Suppose $(G, H)$ is a Gel'fand pair; then since the uniform distribution on $H$ is an element of $\mathcal{B}$, for any $\sigma \in \widehat{G}$ the projection operator $\pi^{\sigma}=|H|^{-1} \sum_{h \in H} \sigma(h)$ has rank at most one. Since this is also true of its conjugates $\pi_{g}^{\sigma}=\sigma(g)^{-1} \pi^{\sigma} \sigma(g)$, we see that $\left(G, H^{g}\right)$ is Gel'fand for all $g \in G$.

As stated above, Bacon, Childs and van Dam [1] showed that the pretty good measurement is optimal for the dihedral groups $D_{n}$ when the hidden subgroup $H$ is of order 2. Indeed, $\left(D_{n}, H\right)$ is Gel'fand for these subgroups, and we generalize their result as follows.

Theorem 5. For any number of registers $k>0$, given the family $\left\{\boldsymbol{\rho}_{g}\right\}$ of density matrices corresponding to random left cosets of conjugate subgroups $\left(H^{g}\right)^{k} \subset G^{k}$ with the uniform distribution on $g$, the pretty good measurement $\left\{E_{g}\right\}$ optimizes the probability of correctly measuring $g$.

Proof. As before, we will show that the optimality conditions hold in each irreducible block, since the $\boldsymbol{\rho}_{g}$, and therefore $M$ and the $E_{g}$, are block-diagonal. Since the tensor product of rank-one operators has rank one, given $\boldsymbol{\sigma}=\sigma_{1} \otimes \cdots \otimes \sigma_{k}$ with $\sigma_{i} \in \widehat{G}$ for all $i$, from (3.1) we have either $\boldsymbol{\rho}_{g}^{\boldsymbol{\sigma}}=0$ or

$$
\boldsymbol{\rho}_{g}^{\boldsymbol{\sigma}}=\left|v_{g}\right\rangle\left\langle v_{g}\right| \otimes \mathbb{1}_{d_{\sigma}}
$$

for some vector $v_{g} \in \boldsymbol{\sigma}$. In the latter case we have $M^{\boldsymbol{\sigma}}=m \otimes \mathbb{1}_{d_{\sigma}}$ where

$$
m=\frac{1}{|G|}\left(\sum_{g}\left|v_{g}\right\rangle\left\langle v_{g}\right|\right)
$$


and

$$
\boldsymbol{\rho}_{g}^{\boldsymbol{\sigma}} E_{g}^{\boldsymbol{\sigma}}=\frac{1}{|G|}\left(\left|v_{g}\right\rangle\left\langle v_{g}\left|m^{-1 / 2}\right| v_{g}\right\rangle\left\langle v_{g}\right| m^{-1 / 2}\right) \otimes \mathbb{1}_{d_{\boldsymbol{\sigma}}}=\frac{C}{|G|} \boldsymbol{\rho}_{g}^{\boldsymbol{\sigma}}\left(M^{\boldsymbol{\sigma}}\right)^{-1 / 2}
$$

where $C$ is defined as the inner product

$$
C=\left\langle v_{g}\left|m^{-1 / 2}\right| v_{g}\right\rangle
$$

Similarly, $E_{g}^{\boldsymbol{\sigma}} \boldsymbol{\rho}_{g}^{\boldsymbol{\sigma}}=\frac{C}{|G|}\left(M^{\boldsymbol{\sigma}}\right)^{-1 / 2} \boldsymbol{\rho}_{g}^{\boldsymbol{\sigma}}$.

It is easy to see that $C$ does not depend on $g$ : if $R_{g}$ denotes the unitary operator corresponding to right multiplication by the diagonal element $(g, \ldots, g)$ in $\mathbb{C}\left[G^{k}\right]$, then $\left|v_{g}\right\rangle=R_{g}\left|v_{1}\right\rangle$ and $m$ commutes with $R_{g}$. Thus $C=\left\langle v_{g}\left|m^{-1 / 2}\right| v_{g}\right\rangle=\left\langle v_{1}\left|R_{g} m^{-1 / 2} R_{g}^{\dagger}\right| v_{1}\right\rangle=\left\langle v_{1}\left|m^{-1 / 2}\right| v_{1}\right\rangle$. Then we have

$$
\sum_{g} \boldsymbol{\rho}_{g}^{\boldsymbol{\sigma}} E_{g}^{\boldsymbol{\sigma}}=\frac{C}{|G|} \sum_{g} \boldsymbol{\rho}_{g}^{\boldsymbol{\sigma}}\left(M^{\boldsymbol{\sigma}}\right)^{-1 / 2}=C\left(M^{\boldsymbol{\sigma}}\right)^{1 / 2}=\frac{C}{|G|} \sum_{g}\left(M^{\boldsymbol{\sigma}}\right)^{-1 / 2} \boldsymbol{\rho}_{g}^{\boldsymbol{\sigma}}=\sum_{g} E_{g}^{\boldsymbol{\sigma}} \boldsymbol{\rho}_{g}^{\boldsymbol{\sigma}}
$$

confirming (1.5).

As for condition (1.6), recalling the equality $\sum_{g} \rho_{g}^{\sigma} E_{g}^{\sigma}=C\left(M^{\sigma}\right)^{1 / 2}$ just above, (1.6) is equivalent to the condition $C\left(M^{\sigma}\right)^{1 / 2} \geq \boldsymbol{\rho}_{g}^{\sigma}$, and hence to $C m^{1 / 2} \geq\left|v_{g}\right\rangle\left\langle v_{g}\right|$. Furthermore, this holds if and only if

$$
\left\langle v_{g}\left|C m^{1 / 2}\right| v_{g}\right\rangle \geq\left\langle v_{g} \mid v_{g}\right\rangle\left\langle v_{g} \mid v_{g}\right\rangle=\left\|v_{g}\right\|^{4}
$$

or, expanding the definition of $C$,

$$
\left\langle v_{g}\left|m^{1 / 2}\right| v_{g}\right\rangle\left\langle v_{g}\left|m^{-1 / 2}\right| v_{g}\right\rangle \geq\left\|v_{g}\right\|^{4} .
$$

We remark that if a positive semidefinite operator $A$ is a linear combination $A=\sum_{\alpha} a_{\alpha} B_{\alpha}$ of positive semidefinite operators $B_{\alpha}$ with $a_{\alpha} \in \mathbb{R}^{+}$, then the kernel of $A$ is $\bigcap_{\alpha} \operatorname{ker} B_{\alpha}$. As $m$ is such a linear combination of operators $\left|v_{g}\right\rangle\left\langle v_{g}\right|$, it follows that $\left|v_{g}\right\rangle$ is orthogonal to ker $m$, and therefore lies in the image of $m$. Thus we can regard $m^{1 / 2}$ and $m^{-1 / 2}$ as inverses, and the inequality (3.2) follows from the following claim:

Claim 6. Let $A$ be a positive operator on a finite dimensional Hilbert space $V$ and let $|v\rangle \in V$. Then

$$
\langle v|A| v\rangle\left\langle v\left|A^{-1}\right| v\right\rangle \geq\|v\|^{4} .
$$

Proof. For a vector $w=\left(w_{1}, \ldots, w_{n}\right)$ of non-negative weights and a real number $r$, define

$$
\mathcal{M}_{r}^{w}\left(x_{1}, \ldots, x_{n}\right)=\left(\frac{\sum_{i} w_{i} x_{i}^{r}}{\sum_{i} w_{i}}\right)^{1 / r}
$$

to be the weighted $r$-mean of the positive vector $x=\left(x_{1}, \ldots, x_{n}\right)$. The power mean inequality $(\mathrm{cf}$. $[8, \S 2.9])$ asserts that for $r<s$ we have $\mathcal{M}_{r}^{w}(x) \leq \mathcal{M}_{s}^{w}(x)$.

The claim follows immediately from the from power mean inequality with $r=-1$ and $s=1$. Specifically, let $B=\left\{\left|b_{i}\right\rangle\right\}$ be a spectral resolution of $A$, so that $B$ is an orthogonal basis of eigenvectors for $V$, and let $\lambda_{i}>0$ be the associated eigenvalues, so that $\lambda_{i}\left|b_{i}\right\rangle=A\left|b_{i}\right\rangle$. Writing $|v\rangle=\sum_{i} v_{i}\left|b_{i}\right\rangle$, we have

$$
\langle v|A| v\rangle=\sum_{i}\left|v_{i}\right|^{2} \lambda_{i} \quad \text { and } \quad\left\langle v\left|A^{-1}\right| v\right\rangle=\sum_{i}\left|v_{i}\right|^{2} \lambda_{i}^{-1} .
$$

If we adopt the weights $w_{i}=\left|v_{i}\right|^{2}$, then $\sum_{i} w_{i}=\|v\|^{2}$ and the claim is equivalent to the the power mean inequality $\mathcal{M}_{-1}^{w}\left(\lambda_{1}, \ldots, \lambda_{n}\right) \leq \mathcal{M}_{1}^{w}\left(\lambda_{1}, \ldots, \lambda_{n}\right)$. 
Returning to the proof of Theorem 5, it remains to establish (1.4). Consider

$$
\begin{aligned}
\left(\sum_{g} \boldsymbol{\rho}_{g}^{\boldsymbol{\sigma}} E_{g}^{\boldsymbol{\sigma}}-\boldsymbol{\rho}_{h}^{\boldsymbol{\sigma}}\right) E_{h}^{\boldsymbol{\sigma}} & =\frac{1}{|G|}\left[\left(C m^{1 / 2}-\left|v_{h}\right\rangle\left\langle v_{h}\right|\right) m^{-1 / 2}\left|v_{h}\right\rangle\left\langle v_{h}\right| m^{-1 / 2}\right] \otimes \mathbb{1}_{d_{\boldsymbol{\sigma}}} \\
& =\frac{C}{|G|}\left[(\Pi-\mathbb{1})\left|v_{h}\right\rangle\left\langle v_{h}\right| m^{-1 / 2}\right] \otimes \mathbb{1}_{d_{\boldsymbol{\sigma}}}
\end{aligned}
$$

where $\Pi$ is the projection operator onto the image of $m$. Since $\left|v_{h}\right\rangle$ lies in this image as discussed above, we have $\Pi\left|v_{h}\right\rangle=\left|v_{h}\right\rangle$ and (3.3) is identically zero.

As in the one-register case, we can distinguish the trivial subgroup from the conjugates of $H$ by completing the PGM with a measurement $M_{0}$ that projects onto the complement of the image of $M$.

\subsection{The Probability of Success}

In this section we give an upper bound on the success probability of the optimal multiregister experiment for Gel'fand pairs. To prepare for this, we record a version of Holevo's theorem on the capacity of a quantum channel [10].

Lemma 7. Let $R=\left\{\rho_{i} \mid i \in I\right\}$ be a family of density matrices treated as linear operators on the Hilbert space $V$. Let $E=\left\{E_{i}\right\}$ be a family of measurement operators on $V$ for which $\sum_{i} E_{i}=\mathbb{1}$ and, for each $i \in I$, $E_{i}$ is a scalar multiple of a projection operator of rank $r$. Then

$$
\operatorname{Exp}_{i} \operatorname{tr} E_{i} \rho_{i} \leq \frac{\operatorname{dim} H}{r|I|}
$$

where the index $i$ is chosen uniformly at random in $I$.

Proof. By assumption, we may write $E_{i}=\alpha_{i} \Pi_{i}$ where $\Pi_{i}$ is a projection operator of rank $r$ and $\alpha_{i} \geq 0$. As $\rho_{i}$ is a density matrix, we may write $\rho_{i}=\sum_{j} p_{j}\left|v_{j}\right\rangle\left\langle v_{j}\right|$ where each $\left|v_{j}\right\rangle$ has unit length, each $p_{j} \in[0,1]$, and $\sum_{j} p_{j}=1$. Observe that

$$
\operatorname{tr} E_{i} \rho_{i}=\sum_{j} p_{j}\left\langle v_{j}\left|E_{i}\right| v_{j}\right\rangle \leq \sum_{j} p_{j}\left\|E_{i}\right\|=\left\|E_{i}\right\|=\alpha_{i}
$$

where $\|A\|$ is the operator norm of $A$, given by $\|A\|=\max _{\mathbf{v} \neq 0}\|A \mathbf{v}\| /\|\mathbf{v}\|$. Hence

$$
\operatorname{Exp}_{i} \operatorname{tr} E_{i} \rho_{i} \leq \frac{\sum_{i} \alpha_{i}}{|I|}
$$

Observe now that $\sum_{i} E_{i}=\mathbb{1}$ and hence that $\operatorname{dim} V=\operatorname{tr} \mathbb{1}=\operatorname{tr} \sum_{i} E_{i}=\sum_{i} \alpha_{i} r ;$ evidently $\sum_{i} \alpha_{i}=\operatorname{dim} V / r$, which completes the proof.

Theorem 8. Let $H$ be a subgroup of $G$ for which $(G, H)$ is a Gel'fand pair and let $H_{G}=\cap_{g} H^{g}$ be the largest normal subgroup contained in $H$. The probability that the optimal k-register measurement correctly identifies a uniformly random conjugate of $H$ is

$$
P_{\text {success }} \leq \frac{1}{|C(H)|} \cdot\left(\frac{|H|}{\left|H_{G}\right|}\right)^{k}
$$

Proof. Without sacrificing optimality, we may initially carry out weak Fourier sampling, in which we observe a representation $\boldsymbol{\sigma}$ with probability

$$
P(\sigma)=\frac{|H|^{k} d_{\boldsymbol{\sigma}} \mathbf{r k} \pi_{g}^{\boldsymbol{\sigma}}}{|G|^{k}}
$$


Again accounting for the fact that $\boldsymbol{\rho}_{g}=\boldsymbol{\rho}_{g^{\prime}}$ when $H^{g}=H^{g^{\prime}}$, the probability of success may then be written

$$
P_{\text {success }}=|\operatorname{Norm}(H)| \cdot \operatorname{Exp}_{g} \operatorname{tr} E_{g} \boldsymbol{\rho}_{g}=|\operatorname{Norm}(H)| \cdot \operatorname{Exp}_{g} \operatorname{Exp}_{\boldsymbol{\sigma}} a_{\boldsymbol{\sigma}} \operatorname{tr} E_{g}^{\boldsymbol{\sigma}} \boldsymbol{\rho}_{g}^{\boldsymbol{\sigma}}
$$

where $\boldsymbol{\sigma}$ is distributed according to $P(\boldsymbol{\sigma}), g$ is uniform in $G$, and $a_{\boldsymbol{\sigma}}=1 / \operatorname{tr} \boldsymbol{\rho}_{g}^{\sigma}$ normalizes $\boldsymbol{\rho}_{g}^{\boldsymbol{\sigma}}$ so that it is a density matrix.

Recall that $\boldsymbol{\rho}_{g}^{\boldsymbol{\sigma}}$ is proportional to $\pi_{g}^{\boldsymbol{\sigma}} \otimes \mathbb{1}_{d_{\boldsymbol{\sigma}}}$ where $\pi_{g}^{\boldsymbol{\sigma}}$ has rank one (or zero), that $M^{\boldsymbol{\sigma}}=m \otimes \mathbb{1}_{d_{\boldsymbol{\sigma}}}$, and that the image of $\pi_{g}^{\sigma}$ is contained in the image of $m$. Therefore, $m^{-1 / 2} \pi_{g}^{\sigma} m^{-1 / 2}$ has rank one or zero, and so $E_{g}^{\sigma}=m^{-1 / 2} \pi_{g}^{\sigma} m^{-1 / 2} \otimes \mathbb{1}_{d_{\boldsymbol{\sigma}}}$ is either zero or a scalar multiple of a projection operator of rank $d_{\boldsymbol{\sigma}}$. By the lemma above, for a representation $\boldsymbol{\sigma}$ we have $\operatorname{Exp}_{g} \operatorname{tr}\left(a_{\boldsymbol{\sigma}} E_{g}^{\boldsymbol{\sigma}} \boldsymbol{\rho}_{g}^{\boldsymbol{\sigma}}\right) \leq d_{\boldsymbol{\sigma}} /|G|$.

Let us again define $S_{H^{k}} \subset \widehat{G^{k}}$ to be the set of representations for which $\pi_{g}^{\sigma}$ is nonzero. By commuting the two expectations above, we conclude that

$$
P_{\text {success }} \leq|\operatorname{Norm}(H)| \operatorname{Exp}_{\boldsymbol{\sigma}} \frac{d_{\boldsymbol{\sigma}}}{|G|}=\frac{|\operatorname{Norm}(H)|}{|G|}|H|^{k} \sum_{\boldsymbol{\sigma}} \mathbf{r k} \pi^{\boldsymbol{\sigma}} \frac{d_{\boldsymbol{\sigma}}^{2}}{|G|^{k}}=\frac{|H|^{k}}{|C(H)|} \operatorname{Planch}\left(S_{H^{k}}\right)
$$

where $\operatorname{Planch}_{G^{k}}(\boldsymbol{\sigma})=d_{\boldsymbol{\sigma}}^{2} /|G|^{k}$ is the Plancherel distribution on $\widehat{G^{k}}$. But this is just the product of the Plancherel distribution on $\widehat{G}$ over the $\sigma_{i}$, so we have

$$
P_{\text {success }} \leq \frac{|H|^{k}}{|C(H)|} \operatorname{Planch}\left(S_{H}\right)^{k}
$$

and recalling from the proof of Theorem 4 that $\operatorname{Planch}\left(S_{H}\right) \leq 1 /\left|H_{G}\right|$ completes the proof.

\subsection{Examples}

Theorem 5 applies to a number of group families that have appeared in the literature on the hidden subgroup problem. Here is a short list of examples of Gel'fand pairs:

- $(G, H)$ where $H$ is normal and $G / H$ is Abelian. Of course, whenever $H$ is normal the hidden conjugate problem becomes trivial.

- $\left(D_{n}, H\right)$ where $H$ consists of the identity and an involution, as in Bacon, Childs and van Dam [1].

- $\left(A_{p}, \mathbb{Z}_{p}^{*}\right)$ where $A_{p}$ is the affine group $\mathbb{Z}_{p}^{*} \ltimes \mathbb{Z}_{p}$ and $\mathbb{Z}_{p}^{*}$ is a maximal non-normal subgroup. An efficient quantum algorithm for the hidden conjugate problem in this case was given by [16].

- All the subgroups of the Heisenberg group, for which an information-theoretic reconstruction algorithm was given by Radhakrishnan, Rötteler and Sen [17].

- $\left(S L_{2}(q), B\right)$ or $\left(G L_{2}(q), B\right)$ where $B$ is the Borel subgroup consisting of upper-triangular matrices.

- $\left(S_{n}, H\right)$ where $H$ is the hyperoctahedral group; this is the centralizer of $(12)(34) \cdots(n-1 n)$, or equivalently the wreath product $S_{n / 2} \prec \mathbb{Z}_{2}$, or the symmetry group of the $(n / 2)$-dimensional hyperoctahedron.

- $\left(S_{n}, H\right)$ where $H=S_{m} \times S_{n-m}$ for some $0 \leq m \leq n$, i.e., the subgroup of permutations under which the set consisting of the first $m$ elements is invariant.

Note there is an efficient classical algorithm for the hidden conjugate problem for the last two examples in $S_{n}$ : simply check for all $\left(\begin{array}{c}n \\ 2\end{array}\right)$ transpositions whether the oracle differs from its value on the identity. This allows us to determine the conjugate of $H$, which is associated with a matching (for the hyperoctahedral group) or a subset of size $m$ (for $S_{m} \times S_{n}$ ). 


\section{Conclusion}

The hidden conjugate problem has important applications; in the dihedral group it is related to hidden shift problems [3] and lattice problems [18]. However, for problems such as Graph Isomorphism, we are typically interested in distinguishing one conjugacy class from another. While we can detect the trivial subgroup with the additional measurement $M_{0}$ defined here and in [1], the PGM is not generally optimal in this case [D. Bacon, personal communication]. Constructing the optimal measurement for the hidden subgroup problem, given a prior on the conjugacy classes, remains an important open question.

\section{Acknowledgments.}

This work was supported by NSF grants CCR-0093065, PHY-0200909, EIA-0218443, EIA-0218563, CCR0220070, and CCR-0220264. We are grateful to David Bacon, Andrew Childs, and Wim van Dam for introducing us to the Pretty Good Measurement and alerting us to reference [22], to the organizers of QIP 2005 at which much of this work was done, to Dan Rockmore and Martin Rötteler for thoughts on Gel'fand pairs, and to Tracy Conrad and Sally Milius for their support and tolerance. C.M. also thanks Rosemary Moore for her recent arrival, and for providing a larger perspective.

\section{References}

[1] David Bacon, Andrew Childs, and Wim van Dam. Optimal measurements for the dihedral hidden subgroup problem. Preprint, quant-ph/0501044 (2005).

[2] David Bacon, Andrew Childs, and Wim van Dam. From optimal measurement to efficient quantum algorithms for the hidden subgroup problem over semidirect product groups. Preprint, quant-ph/0504083 (2005).

[3] Wim van Dam, Sean Hallgren, and Lawrence Ip. Quantum algorithms for some hidden shift problems. Proc. 14th ACM-SIAM Symposium on Discrete Algorithms, pages 489-498, 2003.

[4] Y. C. Eldar, A. Megretski, and G. C. Verghese. Optimal detection of symmetric mixed quantum states. Preprint, quant-ph/0211111.

[5] Mark Ettinger and Peter Høyer. On quantum algorithms for noncommutative hidden subgroups. Preprint, quant-ph/9807029 (1998).

[6] William Fulton and Joe Harris. Representation Theory: A First Course. Number 129 in Graduate Texts in Mathematics. Springer-Verlag, 1991.

[7] P. Hausladen and W. K. Wooters. A 'pretty good' measurement for distinguishing quantum states. J. Mod. Opt. 41, 2385 (1994).

[8] G. Hardy, J. Littlewood, G. Pólya. Inequalities. Cambridge Mathematical Library. Cambridge University Press, 1952.

[9] A. S. Holevo. Statistical decisions in quantum theory. J. Multivar. Anal. 3, 337 (1973).

[10] A. S. Holevo. Some estimates of the information transmitted by quantum communication channels. Problems of Information Transmission 9:177-183, 1973. English translation.

[11] Lawrence Ip. Shor's algorithm is optimal. Preprint, 2004.

[12] Julia Kempe and Aner Shalev. The hidden subgroup problem and permutation group theory. Preprint, quant-ph/0406046 (2004). 
[13] Greg Kuperberg. A subexponential-time quantum algorithm for the dihedral hidden subgroup problem. Preprint, quant-ph/0302112 (2003).

[14] Cristopher Moore, Alexander Russell, and Leonard Schulman. The symmetric group defies strong Fourier sampling: Part I. Preprint, quant-ph-0501056.

[15] Cristopher Moore and Alexander Russell. The symmetric group defies strong Fourier sampling: Part II. Preprint, quant-ph-0501066.

[16] Cristopher Moore, Daniel Rockmore, Alexander Russell, and Leonard Schulman. The value of basis selection in Fourier sampling: hidden subgroup problems for affine groups. Proc. 15th Annual ACMSIAM Symposium on Discrete Algorithms, pages 1113-1122, 2004.

[17] Jaikumar Radhakrishnan, Martin Rötteler, and Pranab Sen. On the Power of Random Bases in Fourier Sampling: Hidden Subgroup Problem in the Heisenberg Groups. Preprint, 2005.

[18] Oded Regev. Quantum computation and lattice problems. Proc. 43rd Symposium on Foundations of Computer Science, pages 520-530, 2002.

[19] Yuval Roichman. Upper bound on the characters of the symmetric groups. Inventiones Mathematicae, 125:451-485, 1996.

[20] Jean-Pierre Serre. Linear Representations of Finite Groups. Number 42 in Graduate Texts in Mathematics. Springer-Verlag, 1977.

[21] Audrey Terras. Fourier analysis on finite groups and applications. Cambridge University Press, 1999.

[22] H. P. Yuen, R. S. Kennedy, and M. Lax. Optimum testing of multiple hypotheses in quantum detection theory. IEEE Trans. Inform. Theory 21:125-134, 1975. 